\title{
Equilíbrio acidobásico, parâmetros urinários e sanguíneos de gatos induzidos ao estresse e suplementados com composto antioxidante
}

\author{
[Acid-base balance, urinary and blood parameters of cats stress-induced cats and \\ supplemented with antioxidant compound] \\ R.C.S. Ogoshi ${ }^{1}$, M.G. Zangeronimo ${ }^{1}$, J.S. Reis ${ }^{1}$, R.V. Sousa ${ }^{1}$, T.M. Gonçalves ${ }^{1}$, K.G. Lisenko $^{1}$, \\ I.O. Alves ${ }^{1}$, K.W. Silva ${ }^{1}$, J. França ${ }^{2}$, F.M.O.B. Saad ${ }^{1 *}$ \\ ${ }^{1}$ Universidade Federal de Lavras - UFLA - Lavras, MG \\ ${ }^{2}$ Faculdade de Medicina Veterinária - Universidade Federal de Uberlândia - UFU - Uberlândia, MG
}

\section{RESUMO}

Este estudo teve como objetivo avaliar a suplementação do composto antioxidante comercial EconomasE (Alltech, Brasil - AOX) sobre o equilíbrio acidobásico, os parâmetros urinários, o hemograma completo e a fragilidade osmótica de eritrócitos (FOE) de gatos estressados. Foram utilizados 24 gatos adultos $(3,49 \pm 0,87 \mathrm{~kg})$, distribuídos em delineamento inteiramente ao acaso, com quatro níveis $(0,250,500$, $750 \mathrm{mg}$ de $\mathrm{AOX} / \mathrm{kg}$ de alimento na matéria seca) e seis repetições, durante o período de 80 dias. Os gatos foram induzidos ao estresse por meio da presença de cães próximo ao recinto experimental (agente estressor; $\mathrm{AE})$, do $61^{\circ}$ dia até o final do experimento. A pressão parcial de dióxido de carbono $\left(\mathrm{pCO}_{2}\right)$ e o bicarbonato $\left(\mathrm{HCO}_{3}^{-}\right)$aumentaram linearmente $(\mathrm{P}<0,05)$ conforme o aumento da ingestão de AOX. Os maiores valores de dióxido de carbono total $\left(\mathrm{tCO}_{2}\right)(\mathrm{P}<0,05)$ foram observados nos gatos alimentados com 500 e $750 \mathrm{mg}$ de AOX/kg de dieta. As concentrações de hemoglobina foram maiores nos animais alimentados com 250 e 500mg de AOX/kg de dieta. Os parâmetros urinários e da FOE foram semelhantes nos gatos em todas as dietas. Esses dados indicam que a suplementação com AOX apresenta efeitos benéficos no equilíbrio acidobásico e na concentração de hemoglobina de gatos induzidos ao estresse.

Palavras-chave: farinha de algas, felinos, gases sanguíneos, selênio

\begin{abstract}
This study aimed to evaluate the supplementation of a commercial antioxidant EconomasE (Alltech, Brazil - AOX) on the acid-base balance, urinary parameters, red blood count (RBC) and erythrocyte osmotic fragility (EOF) of stressed cats. Twenty-four adult cats $(3.49 \pm 0.87 \mathrm{~kg})$ were distributed in a completely randomized design with four levels $(0,250,500,750 \mathrm{mg}$ AOX/kg feed dry matter) and six replicates for a period of 80 days. Cats were induced to stress through the presence of dogs in the experimental environment (stressor agent; AE) from the 61th day to the end. Partial pressure of carbon dioxide $\left(\mathrm{pCO}_{2}\right)$ and bicarbonate $\left(\mathrm{HCO}_{3}\right)$ increased linearly $(\mathrm{P}<0.05)$ with increased intake of AOX. The highest values of total carbon dioxide $\left(t_{C O}\right)(P<0.05)$ were observed in cats fed the 500 and $750 \mathrm{mg}$ AOX/kg diet. Hemoglobin concentration was higher $(P<0.05)$ in animals fed the 250 and $500 \mathrm{mg} \mathrm{AOX/ \textrm {kg }}$ diet. The urinary parameters and EOF were similar among all diets. These data indicate that AOX supplementation has beneficial effects in acid-base balance and hemoglobin concentration of stressinduced cats.
\end{abstract}

Keywords: algae flour, blood gases, feline, selenium

Recebido em 6 de setembro de 2014

Aceito em 1 de março de 2016

*Autor para correspondência (corresponding author)

E-mail: borgesvet@dzo.ufla.br 


\section{INTRODUÇÃO}

Os gatos são animais muito susceptíveis ao estresse (Griffin e Humme, 2006), o que pode resultar em importantes alterações fisiológicas (Carlstead et al., 1993). Assim, muitas vezes torna-se necessário lançar mão de estratégias para conter os efeitos colaterais do estresse, e a suplementação com antioxidantes, nesse caso, pode ser um tratamento eficaz. Os antioxidantes atuam no combate das espécies reativas de oxigênio, produzidas excessivamente durante a situação de estresse, e contribuem na estabilização da condição saudável do animal (Zicker et al., 2006).

Por outro lado, o equilíbrio acidobásico é um processo fisiológico complexo, regulado tanto por tampões intracelulares e extracelulares quanto pelos sistemas urinário e respiratório (Meyer et al., 1995). É passível de ser influenciado por uma série de fatores internos e externos, incluindo os inerentes ao metabolismo, à dieta e às condições ambientais (Olanrewaju et al., 2007, muito embora o organismo sadio consiga compensar, em curto ou em longo prazo, as possíveis alterações ocorridas.

Tem sido relatado em animais de diversas espécies que as respostas ao estresse estão envolvidas com o balanço acidobásico (AguileraTejero et al., 2000; Parker et al., 2003; Wojtas et al., 2013), bem como com o aumento da demanda de oxigênio (Garcia et al., 2012). Nesse sentido, Sivakumar et al. (2010) observaram que a suplementação com antioxidantes (vitamina $\mathrm{C}$, vitamina $\mathrm{E}$ e selênio) pode resultar em melhora no equilíbrio acidobásico.

Há relatos de que o antioxidante comercial (AOX), composto por um pool de antioxidantes não enzimáticos, proporciona aprimoramento da capacidade antioxidante quando suplementado em dietas de aves (Pierce et al., 2009; Xião et al., 2011). Entretanto, o AOX não foi estudado em gatos, e, sobretudo, ainda não se conhecem as possíveis interferências no status acidobásico e nos demais parâmetros fisiológicos desses animais.
Este trabalho tem por objetivo avaliar os efeitos de um suplemento antioxidante em gatos submetidos ao estresse nos parâmetros hematológicos, urinários e no equilíbrio acidobásico.

\section{MATERIAL E MÉTODOS}

O ensaio foi conduzido no Departamento de Zootecnia da Universidade Federal de Lavras, Minas Gerais, no Centro de Estudo em Nutrição de Animais de Companhia (Cenac), e foi aprovado pela Comissão de Ética no Uso de Animais (Comissões/Permanentes/PRP- UFLA - Protocolo $\left.\mathrm{n}^{\circ} 036 / 11\right)$. A temperatura média foi de $20,50 \pm 2,48^{\circ} \mathrm{C}$, e a umidade relativa de $75,60 \pm 8,64 \%$, no período experimental de 80 dias.

Foram utilizados 24 gatos sadios, com idade de 3,5 anos, sem raça definida, machos e fêmeas, peso médio de $3,49 \pm 0,87 \mathrm{~kg}$, alojados individualmente em gaiolas suspensas com dimensões de $0,8 \times 0,8 \times 1 \mathrm{~m}$ (altura $\mathrm{x}$ profundidade $\mathrm{x}$ largura).

Durante todo o período experimental, os animais receberam água ad libitum e um alimento comercial completo, extrusado e seco para gatos adultos (dieta padrão) (Tab. 1). A quantidade de alimento foi calculada segundo a equação de predição energética de manutenção do National Research Council (Nutrient, 2006), 2006), dada por $100 \mathrm{kcal} \times(\mathrm{PC} \mathrm{em} \mathrm{kg})^{0,67}$.

O AOX (EconomasE®, Alltech, Araucária, Brasil) contém em sua composição farinha de algas marinhas (Schizochytrium sp.), levedura enriquecida com selênio $(1.500 \mathrm{mg}$ de selênio $/ \mathrm{kg}$ de produto), ácido ascórbico e produto de fermentação de Aspergillus niger. $\mathrm{O}$ aditivo foi fornecido nas seguintes proporções: 0, 250, $500 \mathrm{e}$ $750 \mathrm{mg} / \mathrm{kg}$ da dieta padrão na matéria seca (MS). Para cada nível, foram utilizados seis gatos, sendo o animal considerado uma parcela experimental. Os animais receberam os diferentes níveis de $\mathrm{AOX}$ veiculados a $2 \mathrm{~mL}$ de leite, exceto para o nível $0 \mathrm{mg} / \mathrm{kg}$ de $\mathrm{MS}$, que recebeu apenas o leite, como forma de padronizar o procedimento adotado. 
Equilíbrio acidobásico...

Tabela 1. Composição nutricional da dieta padrão ${ }^{1}$

\begin{tabular}{ccc}
\hline Níveis nutricionais & Ração seca $(\mathrm{MN} \%)^{2}$ & Ração seca (MS\%) \\
\hline Umidade & 5,39 & - \\
Proteína bruta & 26,91 & 28,44 \\
Extrato etéreo & 11,06 & 11,69 \\
Matéria fibrosa & 2,83 & 2,99 \\
Matéria mineral & 9,48 & 11,69 \\
Extrativo não nitrogenado & 44,32 & 46,85 \\
EM (kcal/kg) & 3519 \\
\hline Composição básica: farinha de carne e ossos, farinha de vísceras, hidrolisado de frango, gordura animal estabilizada, \\
farelo de trigo, milho integral moído, espinafre em pó (min. 0,5\% -equivalente a 5\% espinafre fresco), sementes de \\
linhaça, cloreto de sódio (sal comum), taurina, ácido fosfórico (min. 0,04\%), antioxidantes, corantes e premix \\
vitamínico mineral. Eventuais substitutivos: farelo de arroz desengordurado, glúten de milho 60, arroz quebrado e \\
farelo de soja. \\
${ }^{2}$ Análise realizada no Laboratório de Pesquisa Animal do Departamento de Zootecnia da Universidade Federal de \\
Lavras, Lavras, MG, Brasil. \\
${ }^{3}$ EM: energia metabolizável, calculada com base nos níveis nutricionais, por meio da equação proposta por Nutrient \\
(2006).
\end{tabular}

O desafio (agente estressor; AE) foi promovido do $61^{\circ}$ ao $80^{\circ}$ dia experimental, por meio da inclusão, próximo à sala de experimentação, de seis cães da raça Labrador, que permaneceram até o final do experimento.

No dia zero e no $80^{\circ}$ dia experimental, foram realizadas coletas de sangue para gasometria e hemograma completo. Para a gasometria, em cada dia houve uma coleta de $1,0 \mathrm{~mL} / \mathrm{animal}$ em jejum (às oito horas) e outra após seis horas da alimentação. As avaliações foram realizadas por meio de um analisador de $\mathrm{pH}$ e de gases sanguíneos (Modelo AGS22; Drake Ltda.; São Jose do Rio Preto, Brasil). As variáveis mensuradas foram $\mathrm{pH}$, pressão parcial de dióxido de carbono $\left({ }_{\mathrm{p}} \mathrm{CO}_{2}\right)$, concentração de bicarbonato $\left(\mathrm{HCO}_{3}{ }^{-}\right)$, total de dióxido de carbono $\left(\mathrm{tCO}_{2}\right)$ e excesso de bases (EB). Para o resultado do hemograma completo, foi utilizado $1,0 \mathrm{~mL}$ de sangue de cada animal em jejum em cada dia de observação, utilizando-se o EDTA como anticoagulante e os reagentes hematológicos Dialyse - 3CF, Diascatter Pak, Diaton 3 e Diaclenz (Diagon, Belo Horizonte, Brasil).

Para a avaliação da FOE, foi coletado, no $80^{\circ}$ dia experimental, $1,0 \mathrm{~mL}$ de sangue dos gatos em jejum, com heparina sódica como anticoagulante. A metodologia utilizada foi a descrita por Maede e Hata (1975); desse modo adicionou-se $0,05 \mathrm{~mL}$ de sangue a $5,0 \mathrm{~mL}$ de solução de $\mathrm{NaCl}$ com concentrações de 0,1 a $0,9 \%$. O conteúdo de hemoglobina sobrenadante foi determinado por espectrofotometria a $540 \mathrm{~nm}$ (Bel SPECTRO
S05, Bel Engineering®, Monza, Itália), e a solução contida no tubo de $0,9 \%$ foi considerada como branco ou acerto do zero. A porcentagem de hemólise foi calculada com a solução de $0,1 \%$ $\mathrm{NaCl}$ assumindo $100 \%$ de hemólise.

A densidade e o $\mathrm{pH}$ urinário foram avaliados nos três últimos dias do experimento, seguindo o protocolo proposto por Carciofi (2007). O pH urinário foi mensurado usando-se um pHmetro digital (Modelo DM22; Digimed, São Paulo, Brasil) e a densidade foi determinada por meio de um refratômetro portátil (Modelo RTP20ATC; Instrutherm, São Paulo, Brasil).

Todas as análises estatísticas foram realizadas com o auxílio do programa Statistical Analysis System (SAS, 2004). Os dados foram submetidos à análise de covariância, sendo as medidas no tempo zero as covariáveis e, em seguida, à análise de regressão. Entretanto, quando não houve ajuste, as médias foram comparadas pelo teste de SNK a 5\%. Na análise de FOE, para encontrar as concentrações (valores derivativos) em que ocorreram as porcentagens de hemólise de 5, 50 e 95\%, foi realizada a análise de Probit, por meio do procedimento PROC PROBIT do SAS (2004). Os valores derivativos foram comparados pelo teste não paramétrico de Kruskal-Wallis.

\section{RESULTADOS E DISCUSSÃO}

A ingestão das dietas não influenciou $(\mathrm{P}>0,05)$ nos valores de $\mathrm{pH}$ sanguíneo e no excesso de bases (EB) dos gatos, independentemente do 
período de avaliação: jejum ou pós-prandial (Tab. 2). Para o $\mathrm{tCO}_{2}$, as maiores concentrações $(\mathrm{P}<0,05)$ foram obtidas nos níveis de 500 e $750 \mathrm{mg}$ de $\mathrm{AOX} / \mathrm{kg}$ de dieta na matéria seca (MS). As variáveis pressão parcial de dióxido de carbono $\left(\mathrm{pCO}_{2}\right)$ e bicarbonato $\left(\mathrm{HCO}_{3}^{-}\right)$ apresentaram aumento linear nas concentrações $(\mathrm{P}<0,05)$ conforme a ingestão de $\mathrm{AOX}$, porém somente do período de jejum.

Tabela 2. Valores médios dos parâmetros de gasometria de gatos adultos aos 80 dias após suplementação com antioxidante comercial (AOX)

\begin{tabular}{lcccccccc}
\hline & \multicolumn{2}{c}{ Níveis de $\mathrm{AOX}(\mathrm{mg} / \mathrm{kg}$ de MS $)$} & & & & & \\
Parâmetro & 0 & 250 & 500 & 750 & $\mathrm{CV}$ & $\mathrm{P}^{*}$ & $\mathrm{~L}$ & $\mathrm{Q}$ \\
\hline $\mathrm{pH} \mathrm{j}$ & 7,26 & 7,23 & 7,21 & 7,25 & 0,57 & 0,23 & - & - \\
$\mathrm{pH} \mathrm{pp}$ & 7,13 & 7,25 & 7,25 & 7,30 & 0,70 & 0,14 & - & - \\
$\mathrm{pCO}_{2}(\mathrm{mmHg}) \mathrm{j}$ & 24,81 & 25,13 & 29,57 & 28,15 & 10,86 & 0,04 & 0,01 & 0,50 \\
$\mathrm{pCO}_{2}(\mathrm{mmHg}) \mathrm{pp}$ & 21,15 & 22,77 & 22,85 & 21,65 & 10,46 & 0,52 & - & - \\
$\mathrm{HCO}_{3}^{-}(\mathrm{mmol} / \mathrm{L}) \mathrm{j}$ & 11,23 & 10,67 & 11,83 & 12,48 & 9,23 & 0,04 & 0,02 & 0,18 \\
$\mathrm{HCO}_{3}^{-}(\mathrm{mmol} / \mathrm{L}) \mathrm{pp}$ & 10,73 & 10,25 & 10,30 & 10,85 & 9,03 & 0,64 & - & - \\
$\mathrm{tCO}_{2}(\mathrm{mmol} / \mathrm{L}) \mathrm{j}$ & $12,00^{\mathrm{b}}$ & $11,47^{\mathrm{b}}$ & $12,75^{\mathrm{a}}$ & $13,35^{\mathrm{a}}$ & 16,22 & $0,04^{1}$ & $\mathrm{NS}$ & $\mathrm{NS}$ \\
$\mathrm{tCO}_{2}(\mathrm{mmol} / \mathrm{L}) \mathrm{pp}$ & 11,37 & 10,98 & 11,03 & 11,48 & 11,21 & 0,78 & - & - \\
$\mathrm{EB}(\mathrm{mEq} / \mathrm{L}) \mathrm{j}$ & $-13,63$ & $-14,90$ & $-14,60$ & $-12,87$ & 11,80 & 0,36 & - & - \\
$\mathrm{EB}(\mathrm{mEq} / \mathrm{L}) \mathrm{pp}$ & $-12,58$ & $-14,41$ & $-14,47$ & $-12,68$ & 14,06 & 0,20 & - & - \\
\hline
\end{tabular}

*Regressão polinomial $(\mathrm{P}<0,05)$.

pH: potencial de hidrogênio; j: jejum; pp: pós-prandial; $\mathrm{pCO}_{2}$ : pressão parcial de dióxido de carbono; $\mathrm{HCO}_{3}^{-}$: bicarbonato; $\mathrm{tCO}_{2}$ : dióxido de carbono total; EB: excesso de bases; CV: coeficiente de variação; L: linear; Q: quadrático.

${ }^{1}$ Valores em uma mesma linha, seguidos por letras minúsculas idênticas, não diferem entre si ao nível de $5 \%$ pelo teste de SNK.
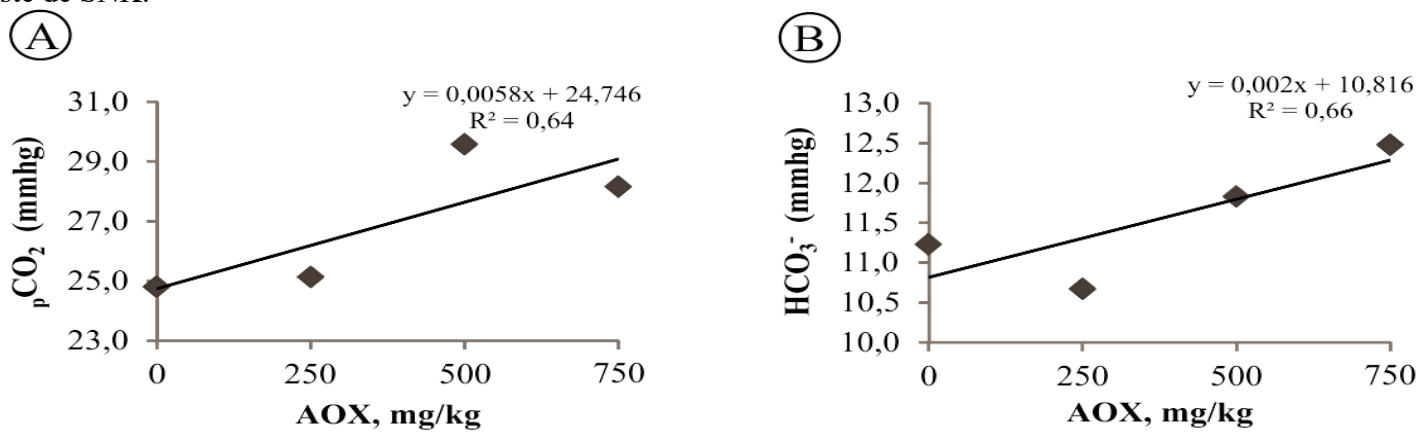

Figura 1. Alterações nas concentrações da pressão parcial de dióxido de carbono (A) e bicarbonato (B) no plasma de gatos em jejum aos 80 dias de suplementação com antioxidante comercial (AOX) mg/kg de matéria seca da dieta.

Embora a hemogasometria em gatos venha sendo estudada em ambos os períodos, jejum e pósprandial (Jeremias et al., 2013; Pires et al., 2013), sabe-se que a metabolização de carboidratos, proteínas e lipídeos resulta em compostos que interferem no equilíbrio acidobásico. Desse modo, a ausência dos resultados das avaliações hemogasométricas pósprandial pode ter sido influenciada pela composição da dieta controle, a qual impediu uma melhor visualização dos efeitos do AOX apenas nesse período. Entretanto, no período em jejum, foram observadas diferenças em alguns parâmetros, demonstrando efeito em longo prazo da ingestão do AOX.

Até o momento, não foram encontrados trabalhos que avaliassem valores hemogasométricos de gatos em situações de estresse quando suplementados com antioxidantes. No entanto, os resultados do presente estudo corroboram os dados encontrados por Sivakumar et al. (2010), que observaram maiores valores de $\mathrm{HCO}_{3}{ }^{-} \mathrm{e}$ $\mathrm{pCO}_{2}$ em cabras estressadas recebendo suplemento antioxidante quando comparadas àquelas estressadas e não suplementadas. Os 
autores consideraram esse efeito como uma melhora no equilíbrio acidobásico.

Além disso, há relatos na literatura (West, 1999; Olanrewaju et al., 2007) de que situações estressantes podem resultar em mudanças na frequência e na profundidade respiratória com consequente expiração excessiva de $\mathrm{CO}_{2}$, resultando na diminuição da $\mathrm{pCO}_{2}$. Portanto, acredita-se que a inclusão de AOX nos maiores níveis atenuou o estresse nos gatos.
Houve diferenças apenas para os níveis de hemoglobina $(\mathrm{P}<0,05) \quad(\mathrm{Tab} .3)$ conforme a ingestão de $\mathrm{AOX}$, em que os maiores valores foram observados nos animais suplementados com os níveis de 250 e $500 \mathrm{mg}$ de $\mathrm{AOX} / \mathrm{kg}$ de matéria seca, e houve tendência $(\mathrm{P}=0,05)$ para hematócrito e contagem de eritrócitos. As demais variáveis dos parâmetros hematológicos, assim como as médias de $\mathrm{pH}$ e densidade urinária, não apresentaram diferenças significativas $(\mathrm{P}>0,05)$ conforme a suplementação de AOX.

Tabela 3. Valores médios dos parâmetros hematológicos e dos parâmetros urinários de gatos adultos após suplementação com antioxidante comercial (AOX)

\begin{tabular}{llllllc}
\hline \multirow{2}{*}{ Variável } & \multicolumn{7}{c}{ AOX, mg/kg de MS } & & \\
& 0 & 250 & 500 & 750 & $\mathrm{CV}(\%)$ & $\mathrm{P}$ \\
\hline Eritrócitos (milhões/dL) & 8,29 & 9,53 & 9,12 & 8,47 & 8,52 & 0,05 \\
Hematócrito (\%) & 36,63 & 42,18 & 40,63 & 37,91 & 6,26 & 0,05 \\
Hemoglobina (g/dL) & $11,98^{\mathrm{b}}$ & $13,77^{\mathrm{a}}$ & $13,53^{\mathrm{a}}$ & $12,40^{\mathrm{b}}$ & 8,16 & $<0,01$ \\
V.C.M. (fL) & 44,10 & 44,18 & 44,57 & 44,75 & 2,88 & 0,80 \\
C.H.C.M (\%) & 32,73 & 32,72 & 33,27 & 32,67 & 2,15 & 0,63 \\
H.C.M (pg) & 14,43 & 14,45 & 14,82 & 14,62 & 3,16 & 0,46 \\
LT (mil/mm3) & 15,60 & 14,33 & 16,62 & 13,92 & 22,69 & 0,90 \\
Plaquetas (mil/mm3) & 580,17 & 552,17 & 689,33 & 567,83 & 21,90 & 0,32 \\
pH urina & 7,90 & 7,40 & 7,70 & 7,40 & 6,99 & 0,24 \\
Densidade urina & 1025 & 1027 & 1028 & 1021 & 0,84 & 0,54 \\
\hline
\end{tabular}

VCM: volume celular médio dos eritrócitos; CHCM: concentração hemoglobínica corpuscular média; LT: leucócitos totais; CV: coeficiente de variação.

Os eritrócitos são importantes indicadores de estresse oxidativo (Machado et al., 2009), e em animais de companhia, o principal alvo da lesão oxidativa é a hemoglobina (Caldin et al., 2005). As maiores concentrações da hemoglobina nos gatos suplementados com 250 e $500 \mathrm{mg}$ de $\mathrm{AOX} / \mathrm{kg}$ de MS estão de acordo com Sivakumar et al. (2010) e Yousef et al. (2003), que também encontraram o mesmo resultado em caprinos e coelhos estressados suplementados com antioxidantes.

A elevação da hemoglobina sugere algumas hipóteses fisiológicas, e uma delas pode ser devido ao fato de que a hemoglobina corresponde a mais de $80 \%$ da capacidade tampão não bicarbonato presente no sangue (Dibartola, 2006) e pode estar reduzida em situações de desequilíbrio acidobásico (West, 1999). Outra hipótese pode ser em razão de a redução de eritrócitos e, consequentemente, da hemoglobina já ter sido associada à exaustão na capacidade de eritropoiese ocasionada pelo estresse (Garcia et al., 2012). Alguma dessas hipóteses pode ter ocorrido nos animais não suplementados com AOX e, de maneira inesperada, nos que ingeriram 750mg de $\mathrm{AOX} / \mathrm{kg}$ de $\mathrm{MS}$, o que sugere um possível excesso do produto, no entanto um estudo mais amplo dessas respostas deve ser feito para a confirmação.

Quanto aos parâmetros urinários, o pH urinário pode se tornar alcalino ou ácido a partir da compensação devido às alterações do $\mathrm{pH}$ sanguíneo (Dibartola, 2006). No presente trabalho, no entanto, observou-se que o AOX não interfere nesses parâmetros. Não foram encontradas pesquisas que permitissem uma discussão mais ampla dos resultados aqui achados.

As curvas acumulativas (médias) da FOE obtidas após 80 dias de suplementação com AOX de gatos foram semelhantes para todos os níveis (Fig. 2). 


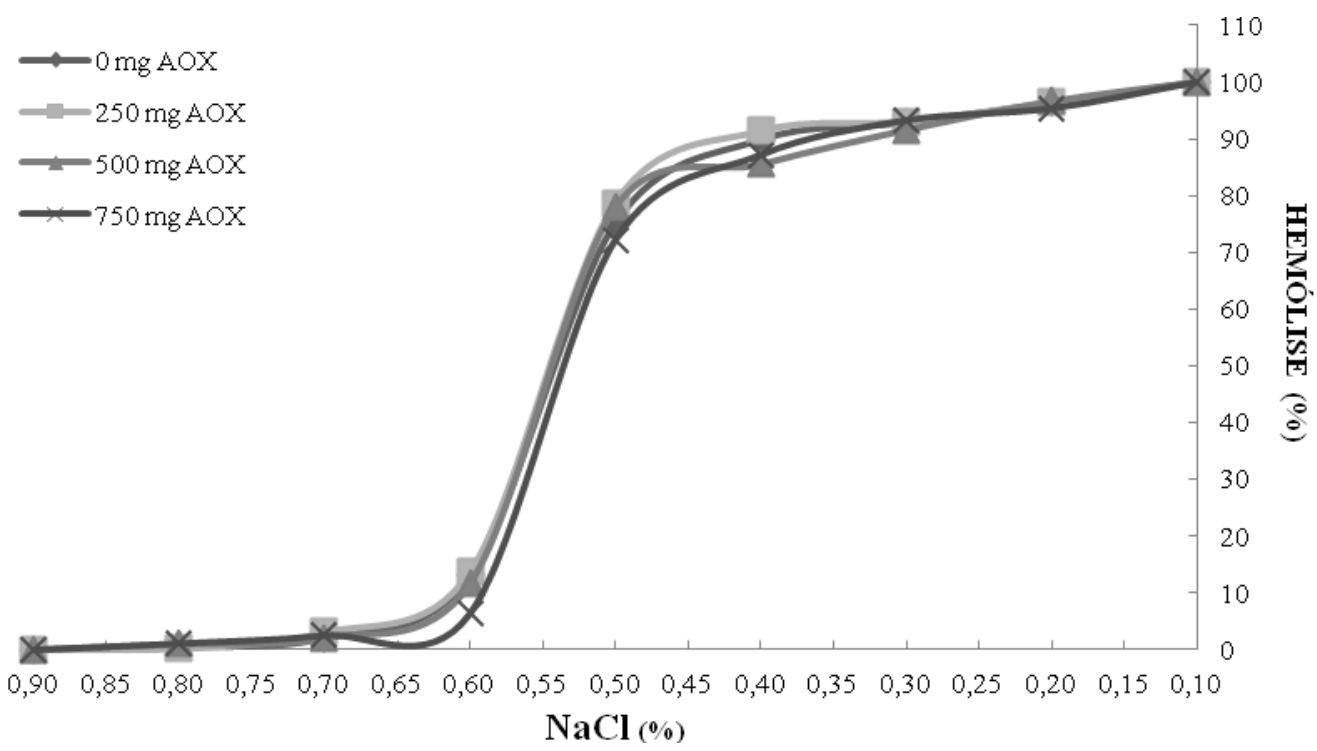

Figura 2. Curvas acumulativas (médias) da fragilidade osmótica eritrocitária de gatos após 80 dias da suplementação com antioxidante comercial (AOX) mg/kg de matéria seca da dieta/kg de MS na dieta.

Não foram observadas diferenças $(\mathrm{P}>0,05)$ na FOE quando avaliados os valores derivativos médios das concentrações de solução salina tamponada que corresponderam a 5, 50 e $95 \%$ de hemólise (Tab. 4). A FOE consiste numa técnica ainda pouco empregada e, portanto, com precárias referências para gatos em circunstâncias diversas. Contudo, os valores das concentrações de solução salina $(\% \mathrm{NaCl})$, em que se observou $50 \%$ de hemólise, apresentaramse semelhantes ao valor médio de 0,54 para gatos sadios, obtido por Jain (1973).

Tabela 4. Valores médios (derivativos) das concentrações de solução salina $(\% \mathrm{NaCl})$ correspondentes a 5,50 e $95 \%$ de hemólise em gatos adultos após 80 dias de suplementação com antioxidante comercial (AOX) $\mathrm{mg} / \mathrm{kg}$ de MS

\begin{tabular}{ccccccc}
\hline & \multicolumn{9}{c}{ AOX } & & P \\
\cline { 2 - 5 } Hemólise & 0 & 250 & 500 & 750 & CV & 0,66 \\
$5 \%$ & 0,87 & 0,88 & 0,89 & 0,89 & 7,36 & 0,64 \\
$95 \%$ & 0,51 & 0,53 & 0,52 & 0,52 & 5,74 & 0,68 \\
\hline
\end{tabular}

*Não significativo pelo teste de Kruskal-Wallis ao nível de 5\%; CV: coeficiente de variação.

O aumento da FOE pode ocorrer em situações de estresse devido ao aumento da peroxidação lipídica, a qual atrapalha a organização dos fosfolipídeos (fosfatidilserina e da fostadiletanolamina) da membrana plasmática (Jain, 1984) e, consequentemente, reduz a fluidez celular (Hebbel, 1986). Sabe-se que o antioxidante ácido gálico protegeu a integridade da membrana dos eritrócitos de ratos diabéticos, apresentando menor porcentagem de hemólise (Ramkumar et al., 2014). Há relato de que a suplementação com vitamina E, outro potente antioxidante, reduziu a FOE em ratos induzidos ao estresse oxidativo (Ambali et al., 2010). Foram atribuídos ao composto antioxidante AOX efeitos fisiológicos redundantes à vitamina $\mathrm{E}$ em aves (Xião et al., 2011). Apesar disso, contrariando esses resultados, o atual trabalho demonstra que a inclusão do AOX pode não ter sido suficiente para alterar a FOE de gatos induzidos ao estresse.

\section{CONCLUSÕES}

A suplementação de AOX em dietas apresenta efeitos benéficos para os parâmetros relacionados ao equilíbrio acidobásico e na concentração de hemoglobina de gatos induzidos ao estresse, sendo recomendada, nas condições estudadas, uma suplementação de até $500 \mathrm{mg}$ de 
$\mathrm{AOX} / \mathrm{kg}$ de matéria seca. Por se tratar de um experimento pioneiro, tornam-se necessários mais estudos para compreensão dos mecanismos de ação do AOX, sobretudo com outros agentes estressores para gatos.

\section{AGRADECIMENTOS}

À empresa Alltech, pelo financiamento do projeto; ao $\mathrm{CNPq}$ pela concessão da bolsa de estudos e à Fapemig pelo apoio financeiro.

\section{REFERÊNCIAS}

AGUILERA-TEJERO, E.; ESTEPA, J.C.; LÓPEZ, I. et al. Quantitative analysis of acidbase balance in show jumpers before and after exercise. Res. Vet. Sci., v.68, p.103-108, 2000.

AMBALI, S.F.; AYO, J.O.; OJO, S.A.; ESIEVO, K.A. Vitamin $\mathrm{E}$ protects rats from chlorpyrifosinduced increased erythrocyte osmotic fragility in Wistar rats. Food Chem. Toxicol., v.48, p.3477-3480, 2010.

CALDIN, M.; CARLI, E.; FURLANELLO, T. et al. A retrospective study of 60 cases of eccentrocytosis in the dog. Vet. Clin. Pathol., v.34, p.224-231, 2005.

CARCIOFI, C. Métodos para estudo das respostas metabólicas de cães e gatos a diferentes alimentos. Rev. Bras. Zootec., v.36, Supl. Esp., p.235-249, 2007.

CARLSTEAD, K.; BROWN, J.L.; STRAWN, W. Behavior and physiological correlates of stress in laboratory cats. Appl. Anim. Behav. Sci., v.38, p.143-158, 1993.

DIBARTOLA, S.P. (Ed.). Fluid, electrolyte and acid-base disorders in small animal practice. 3.ed. Missouri: Elsevier, 2006. 250p.

GARCIA, F.; SCHALCH, S.H.C.; ONAKA, E.M. et al. Hematologia de tilápia-do-nilo alimentada com suplemento a base de algas frente a desafios de estresse agudo e crônico. Arq. Bras. Med. Vet. Zootec., v.64, p.198-204, 2012.

GRIFFIN, B.; HUME, K.R. Recognitions and management of stress in housed cats. In: AUGUST, J.R (Ed.). Consultation in feline internal medicine. St. Louis: Elsevier, 2006. p. 717-727.
HEBBEL, R.P. Erythrocyte antioxidants and membrane vulnerability. J. Lab. Clin. Med., v.107, p.401-404, 1986.

JAIN, N.C. Osmotic fragility of erythrocytes of dogs and cats in health and in certain hematologic disorders. Cornell Vet., v.63, p.411423, 1973.

JAIN, S.K. The accumulation of malonyldialdehyde, a product of fatty acid peroxidation, can disturb aminophospholipid organization in the membrane bilayer of human erythrocytes. J. Biol. Chem., v.259, p.3391-3394, 1984.

JEREMIAS, J.T.; NOGUEIRA, S.P.; BRUNETTO, M.A. Predictive formulas for food base excess and urine $\mathrm{pH}$ estimations of cats. Anim. Feed Sci. Technol., v.182, p.82-92, 2013.

MACHADO, L.P.; KOHAYAGAWA, A.; SAITO, M.E. et al. Lesão oxidativa eritrocitária e mecanismos antioxidantes de interesse na medicina veterinária. Rev. Cienc. Agrovet., v.8, p.84-94, 2009.

MAEDE, Y; HATA, R. Studies on feline haemobartonellosis II. The mechanism of anemia produced by infection with Haemobartonella felis. J. Vet. Med. Sci., v.37, p.49-54, 1975.

MEYER, D.J.; COLES, E.H.; RICH, L.J. Medicina de laboratório veterinário: interpretação e diagnóstico. São Paulo: Roca, 1995. 308p.

NUTRIENT requirements of dogs and cats. Washington: National Academy of Science, 2006. 398p.

OLANREWAJU, H.A.; THAXTON, J.P.; DOZIER, W.A.; BRANTON, S.L. Electrolyte diets, stress, and acid-base balance in broiler chickens. Poult. Sci., v.86, p.1363-1371, 2007.

PARKER, A.J.; HAMLIN, G.P.; COLEMAN, C.J.; FITZPATRICK, L.A. Quantitative analysis of acid-base balance in Bos indicus steers subjected to transportation of long duration. $J$. Anim. Sci., v.81, p.1434-1439, 2003.

PIERCE, J.L.; AO, T.; POWER, R.F. et al. Investigation of replacing vitamin $\mathrm{E}$ with EconomasE® in broiler diet. Poult. Sci,. v.88, Suppl. 1, p.97, 2009. 
PIRES, C.P.; SAAD, F.M.O.B.; OGOSHI, R.C.S. et al. Urinary acidifier in diet with high excess base for adult cats. Cienc. Agrotec., v.37, p.359-368, 2013.

RAMKUMAR, K.M.; VIJAYAKUMAR, R.S.; VANITHA, P. et al. Protective effect of gallic acid on alloxan-induced oxidative stress and osmotic fragility in rats. Hum. Exp. Toxicol., v.33, p.638-649, 2014.

SAS user's guide, version 9.00, 4.ed. Cary: SAS, 2004. 200p.

SIVAKUMAR, A.V.N.; SING, G.; VARSHNEY, V.P. Antioxidants supplementation on acid base balance during heat stress in goats. J. Anim. Sci., v.23, p.14621468, 2010.

WEST, J.W. Nutritional strategies for managing the heat stressed dairy cow. J. Anim. Sci., v.77, suppl. 2, p.21-35, 1999.
WOJTAS, K.; CWYNAR, P.; KOLACZ, R.; et al. Effect of heat stress on acid base balance in Polish Merino sheep. Arch. Tierz., v.56, p.917923, 2013.

XIÃO, R.; POWER, R.F.; MALLONEE, D. et al. comparative transcriptomic study of vitamin $\mathrm{E}$ and an algae-based antioxidant as antioxidative agents: investigation of replacing vitamin $\mathrm{E}$ with the algae-based antioxidant in broiler diets. Poult. Sci., v.90, p.136-146, 2011.

YOUSEF, M.I.; SALEM, M.H.; KAMEL, K.I. et al. Influence of ascorbic acid supplementation on the hematological and clinical biochemistry parameters of male rabbit exposed to aflatoxin B. J. Environ. Sci. Health B., v.38, p.193-209, 2003.

ZICKER, S.C.; WEDEKIND, K.J.; JEWELL. D.E. Antioxidants in veterinary nutrition. Vet. Clin. North Am. Small Anim. Pract., v.36, p.1183-1198, 2006. 